Torus状の Magnetic bottle をつくろうとするとよく知られているよ5に、粒子は driftを弓けて、朝方向汇逃げてしまう。Pla sma 平衡から云えばcharge separa-

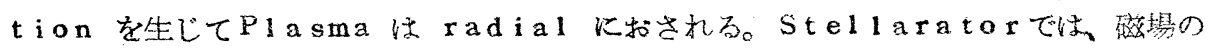
rotat jonal transformでこれを防いでいる。以下には閉じた磁力線でこのdrif 至防ぐ方法をsingle particleの报い方で考えてみる。driftがなくなれば加速器とし して皁くであろうし又、Pyrotron 式のPlasma confinementの方法としても利用 用できるであろう。

\title{
\&1. Drift
}

粒子の磁場中のgyro-radius は砇力線の曲率に比べてずつと小さいと仮定ん粒子の運

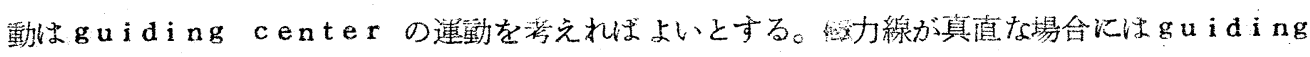

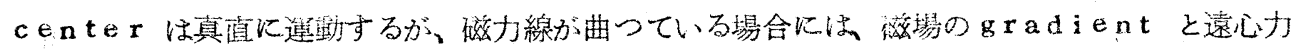
により

このときのdrift velocity はSpitzerの本によれば

$$
W d=\frac{m c}{B}\left(\frac{1}{z} v_{1}^{2}+v_{n}^{2}\right) .
$$

今、s stel1aratorのように粒子群をconfineすることを考光る。ただ、今の場合に

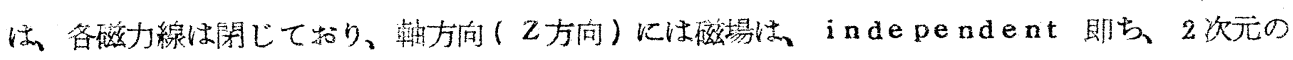

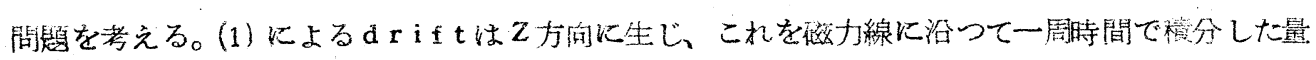
が1国あたりのZ方向への変位であり、これをできるだけ小さくすることを考える。

$$
z_{p}=\int w_{d} d t
$$

磁力線の力向化 1 ine element ds をとれば

$$
d t=\frac{d t}{v_{11}}
$$

(1) (2) (3) から

$$
Z_{D}=\int w_{0} d t=\oint \frac{m c}{e B \rho v_{i 1}}\left(\frac{1}{2} v^{2}+v_{i 1}^{2}\right) d s \quad(1-4)
$$


ゆは閣じた磁力線について一周を意味する。

$$
\begin{aligned}
& v^{2}=v_{i}^{2}+v_{1}^{2} \\
& \frac{1}{2} m v_{1}^{2}=B_{1}
\end{aligned}
$$

であるか、(4)は

そなる。

$$
z_{D}=\int \frac{m c v^{2}-v^{2}}{e B \rho\left(v^{2}-v_{1}^{2} / 2\right.} d s=f \frac{m c\left(v^{2}-B m\right.}{e B g\left(v^{2}-\frac{2 B}{m}\right) / 2} d s
$$

$Z_{\mathrm{D}}=0$ とすることができれ朵 single particle としてのconfinement が できるわけであり、charge.seperation も生じないわけで女る。 ここで(7)を簡単にする。そのためには

$$
v^{2}>r^{2}
$$

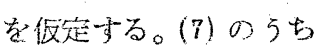

$$
\begin{aligned}
& \text { 7) } 55 \\
& \left(v^{2}-\frac{1}{2} v^{2}\right)\left(v^{2}-v^{2}\right)^{-\frac{1}{2}}=v^{2}\left(1-\frac{1-v_{1}^{2}}{v^{2}}\right)\left(v^{2}\right)^{-\frac{1}{2}}\left(1-\frac{v_{2}^{2}}{v^{2}}\right)^{-\frac{1}{2}} \\
& \fallingdotseq v\left(1-\frac{1}{2} \frac{v^{2}}{v^{2}}\right)\left(1+\frac{v_{1}^{2}}{2 v^{2}}+\frac{z}{9} \frac{v^{4}}{v^{4}}\right)=v\left(1+\frac{1}{8} \frac{v_{2}^{4}}{v^{4}}\right)
\end{aligned}
$$

であるから

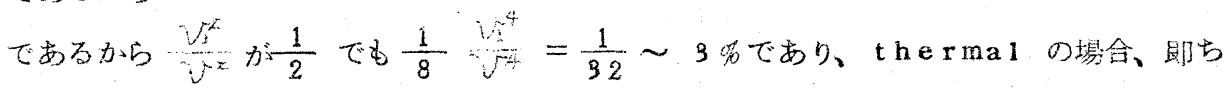

$$
\frac{v^{2}}{\sqrt{x}} \sim \frac{2}{3} \text { のとさ } \frac{1}{8} \times \frac{4}{9}=\frac{1}{18} \sim 5 \% \text { にすこない。 }
$$

侹つて (7) は略

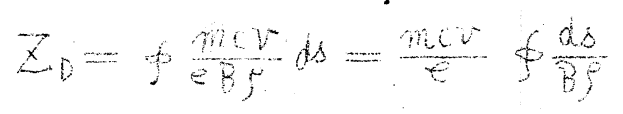

-となる。

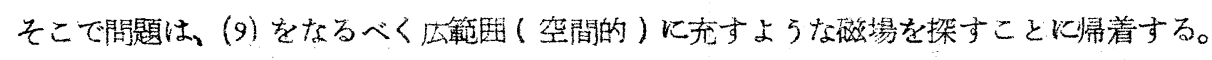

\$2. 磁塕が与えられた場合の drift の計算

（1－9）を磁力線と皎気 potent i a l 線をつかつた座標に移つてかきなおしてみる。 円筒座標をとり、呟場は $\mathbf{Z}$ 成分をもたないとする。 
d i $\overrightarrow{\mathbf{B}}=0$ より

$$
\frac{\partial(r B r)}{\partial r}+\frac{\partial B \phi}{\partial \phi}=0
$$

$\operatorname{rot} \vec{B}=0$ 上り

$(2-1)$ より

$$
\frac{\partial B r}{\partial \phi}-\frac{\partial(r \beta \phi)}{\partial r}=0
$$

$(2-2)$ より

$$
\left\{\begin{array}{l}
r B_{r}=\frac{\partial \psi}{\partial \phi} \\
B_{\phi}=-\frac{\partial \psi}{\partial r}
\end{array}\right.
$$

とかける。

$$
\left\{\begin{array}{l}
B_{r}=\frac{\partial \Omega}{\partial \phi} \\
r B_{\phi}=\frac{\partial \Omega}{\partial r}
\end{array}\right.
$$

= const は磁力線、= constはpotential 線をあらわす。

$$
\left\{\begin{array}{l}
d \psi=\frac{\partial \psi}{\partial r} d r+\frac{\partial \psi}{r \partial \phi} d \phi \\
d \Omega=\frac{\partial \Omega}{\partial r} d r+\frac{\partial \Omega}{r \partial \phi} d \phi
\end{array}\right.
$$

$\mathrm{dv}, \mathrm{rd} \oint$ にいてとけば

$$
\begin{aligned}
& d r=\frac{1}{\Delta}\left(\frac{\partial \Omega}{r \partial \phi} d \psi-\frac{\partial \psi}{r \partial \phi} d \Omega\right) \\
& r d \phi=\frac{1}{\Delta}\left[\frac{\partial \psi}{\partial r} d \Omega-\frac{\partial \Omega}{\partial r} d \psi\right] \\
& \text { ここ } \Delta=\left|\begin{array}{l}
\frac{\partial \psi}{\partial r}, \frac{\partial \psi}{r \partial \phi} \\
\frac{\partial \Omega}{\partial r}, \frac{\partial \Omega}{r \partial \phi}
\end{array}\right|
\end{aligned}
$$

これから 1 ine element

をつくると、(2-3),(2-4)をつかつて

$$
\begin{aligned}
& d s^{2}=\frac{B^{2}}{\Delta^{2}}\left[d \Omega^{2}+d \psi^{2}\right] \\
& d s^{2}=\frac{1}{B^{2}}\left[d \psi^{2}+d \Omega^{2}\right]
\end{aligned}=-B^{2} \text { であるから }
$$




$$
\text { 大 河 } 干 \text { 弘 }
$$

となる、

直交曲線座標 $\mathrm{ds}^{2}=\mathrm{h}_{1}^{2} \mathrm{du}_{1}^{2}+\mathrm{h}_{2}^{2} \mathrm{du}_{2}^{2}$ で $\mathrm{u}_{1}=\mathrm{const}$ の c u r ve の曲率は，岩田氏 によれば

$$
\frac{1}{\rho}=\frac{1}{h_{1} h_{z}} \frac{\partial h_{z}}{\partial u_{1}}
$$

で与えられる。

従つて $\psi=$ const の磁力線の曲率は

$$
\frac{1}{\rho}=B^{2} \frac{\partial}{\partial \psi} \cdot \frac{1}{B}
$$

で与えられる。

又、磁力線に沿つての1 ine el ement は $\frac{\mathrm{d} \Omega}{\mathrm{B}}$ であるから( 1-9)の積分は結局

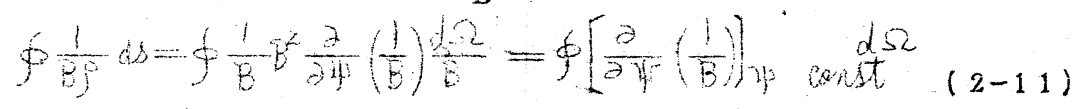

となる。

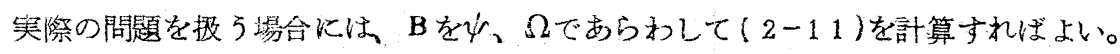
(2ー11)をかきなすと

$$
\frac{\partial}{\partial \psi} \phi \frac{d \Omega}{B}=0
$$

ここで $\frac{\mathrm{d} \Omega}{\mathrm{B}}$ が磁力線に沿つた1 ine element であるから、磁力線の長さが磁力線によつて かわらなければよいことを示している。

\section{§3. Pl asma 平衡との関係}

P1 asmaは充分ウスイとし笔磁場は外部場でけとする。

p 1 asma 平衡の条件は

$$
\overrightarrow{\mathrm{J}} \times \overrightarrow{\mathrm{B}}=\eta^{\mathrm{P}}
$$

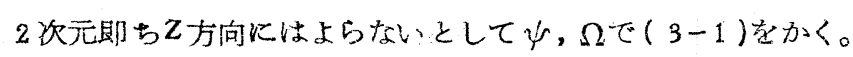

$$
J_{z} \cdot B_{\alpha}=\frac{1}{\frac{1}{b}} \frac{\partial P}{\partial \psi}
$$

B囚は実はBであるから

$$
j_{x}=\frac{\partial P}{\partial \psi}
$$

所謂 charge seperatior が括こらないために住例えばあるZ= const の面で 


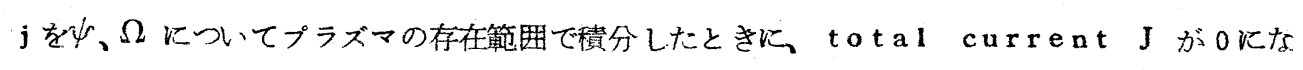
ればよい。

$$
J=\iint z \frac{d \psi}{B} \frac{d \Omega}{B}
$$

$\mathrm{d} \Omega$ Kついての㱴分はせを onstして一周行5。

(アータ)をつからてかきな特せば

$$
J=\iint \frac{\partial P}{\partial \psi} \frac{1}{B_{z}} d \psi d \Omega
$$

pressurépは、ঋたけ即ち、磁力線に直角方向だけの函数

郘与。

$$
P=P(\psi)
$$

とし ( 3-5)を部分穔分すれば

$$
\left.J=\iint P(\psi) \cdot \frac{1}{\beta_{2}} d \Omega\right] \psi-\int P(\psi) \frac{\partial}{\partial \psi}\left(\frac{1}{\beta_{2}}\right) d \psi(3-7)
$$

为一項は、Pがプラズマ外部で0.であることからきえる。

従つて

$$
J=-\int p(\psi)\left(\frac{\partial}{\partial \psi} \int \frac{1}{B^{2}} d \Omega\right) d y \quad(3-8)
$$

となる。

$\mathbf{J}=0$ になるためには

$$
\frac{\partial}{\partial 4} f \frac{d s}{B^{2}}=0
$$

ならばよい。

§ 4. Analytical 計算の例

例として 磁場 $\overrightarrow{\mathrm{B}}$

$$
\vec{B}=\left\{B_{\mathbf{r}} ; B_{\phi}, 0\right\}
$$

をとる。

$$
\left\{\begin{array}{l}
B_{r}=B_{0} \frac{r_{0}}{r}\left(\frac{r}{r_{0}}\right)^{N_{S}} \sin N_{\phi} \\
B_{\phi}=B_{0} \frac{r}{r}\left[1+f\left(\frac{r}{r_{0}}\right)^{N} \operatorname{Cos} N_{\phi}\right]
\end{array}\right.
$$

ঋ， $\Omega$ を計算すると 


$$
\left\{\begin{array}{c}
\text { 大 河 } \mp \text { 弘 } \\
\Psi=-B_{0} r_{0}\left[\ln \frac{r}{r_{0}}+\frac{f}{N}\left(\frac{r}{r_{0}}\right)^{N} \cos N \phi\right] \\
\Omega=B_{0} r_{0}\left[\phi+\frac{f}{N}\left(\frac{N}{r_{0}}\right)^{2} \sin N \phi\right]
\end{array}\right.
$$

r，めを廿，四にかかえねばならない。

簡単にするため、 $\mathrm{B}_{0}, \boldsymbol{r} 0$ を省き更に

と怔く。

$$
\ln \frac{\mathbf{r}}{r_{0}}=-\mu
$$

$$
\left\{\begin{array}{l}
\psi=-\left[-\mu+\frac{f}{N} e^{-N \mu} \cos N \phi\right] \\
\Omega=\phi+\frac{f}{N} e^{-N \mu} \sin N \phi
\end{array}\right.
$$

かきな扎て

$$
\left\{\begin{array}{l}
\mu=\psi+\frac{f}{N} e^{-N u} \cos N \phi \\
\phi=\Omega-\frac{f}{N} e^{-N u} \sin N \phi
\end{array}\right.
$$

ここで $\mathrm{u}+\mathrm{i} \phi=z, \psi+\mathrm{i} \Omega=\zeta$ と怙きかえなおすと

$$
z=5+\frac{f}{N} e^{-N z}
$$

が觉らる。

$\frac{\mathbf{f}}{\mathbf{N}} \ll 1$ と仮定し

\section{Lagrange の遡函数の公式}

$$
\begin{aligned}
& x=\xi+\epsilon \mathrm{F}(x) \text { のを } \\
& \begin{array}{l}
x=\xi+\sum_{n=1}^{\infty} \epsilon^{n} \frac{1}{n !}\left(\frac{d}{d \xi}\right)^{n-1}\{F(\xi)\}^{n}, \cdot(4-8) \\
g(x)=g(\xi)+\sum_{n=1}^{\infty} \epsilon^{n} \frac{1}{n !}\left(\frac{d}{d \xi}\right)^{n-1}\left\{g^{\prime}(\xi)(F(\xi))^{n}\right\}
\end{array} \\
& Z=h+\sum_{n=1}^{\infty}\left(\frac{f}{N}\right)^{n} \frac{1}{n !}\left(\frac{d}{d b}\right)^{n-1}\left(e^{-N_{n} b}\right) \\
& e^{-M Z}=e^{-M n}+\sum_{n=1}^{\infty}\left(\frac{f}{N}\right)^{n} \frac{1}{n !}\left(\frac{d}{d n}\right)^{n-1}\left[-M e^{-(M-n N) !}\right] \quad(4-9) \\
& Z=b+\frac{f}{N} e^{-N t}-\frac{f^{2}}{N} e^{-2 N \hbar}+\cdots \\
& e^{-M E}=e^{-M b}-\frac{M}{N} f e^{-(M+N) t}+\frac{t^{2}}{2} \\
& \frac{M(M+2 N)}{N^{2}} e^{-(M+2 N)}+\cdots \cdot \\
& -573-
\end{aligned}
$$




\section{Toroidal Magnetic bottle}

これらをつかつて

$$
\frac{1}{B}=e^{-\lambda}\left[1+2 f e^{-N \mu} \cos N \phi+f^{2} e^{-2 N \mu}\right]^{-\frac{1}{2}}
$$

をభ、のでかきな拈す。

$$
\begin{aligned}
& e^{-N u} \cos N \phi=\operatorname{Re}\left(e^{-N Z}\right) \\
& =e^{-N 4}\left[\cos N \Omega \cdot f_{e}^{-N \psi} \cos 2 N \Omega \frac{3}{2} f^{2} e^{-3 N 4} \operatorname{Cos} 3 N \Omega\right] \\
& e^{-\mu} \quad=e^{-\psi}\left[1-\frac{f}{N} e^{-N \psi} \cos N \Omega+\frac{f^{2}}{z}\left(\left(\frac{z}{N}+\frac{1}{2 N^{2}}\right) \cos 2 N \Omega\right.\right. \\
& \left.e^{-2 N u}+\frac{1}{2 N^{2}} \mid e^{-2 N}\right] \\
& =e^{-2 N 4}\left[1-2 f e^{-N \psi} \operatorname{Cus} N \Omega+f^{2}(1+3 \cos \angle N \Omega) e^{-2 N 4}\right] \\
& \frac{1}{B} e^{-\psi}\left(1-f\left(1+\frac{1}{N}\right) e^{-N \psi} \cos N \Omega\right. \\
& \left.+f^{2}\left\{\cos 2 N \Omega\left(\frac{3}{2 N}+\frac{1}{4 N^{2}}+\frac{7}{4}\right)+\frac{1}{4}+\frac{1}{2 N}+\frac{1}{4 N^{2}}\right\} e^{(2-12)}\right\}
\end{aligned}
$$

従つて

これから

$$
\begin{aligned}
\frac{1}{2 \pi} \frac{\partial}{\partial \psi} f \frac{d \Omega}{B} & =\frac{\partial}{\partial \psi}\left[e^{-\psi}+f^{2}\left(\frac{1}{4}+\frac{1}{2 N}+\frac{1}{4 N^{2}}\right) e^{-(2 N+1)+4}\right. \\
& =\left[-e^{-4} f^{2}(2 N+1)\left(\frac{1}{4}+\frac{1}{2 N}+\frac{1}{4 N}\right) e^{-(2 N+1) \psi}\right]
\end{aligned}
$$

これが至る所 ○になることはづきないが例えばれれ０の䂭力線については充すことができる。 at $\psi=0$

$$
\begin{aligned}
& \quad \frac{1}{2 \pi} \frac{\partial}{\partial \psi} \phi \frac{\alpha \Omega}{B}=\left[-1-f^{2}(z N+1)\left(\frac{1}{4}+\frac{1}{2 N}+\frac{1}{4 N^{2}}\right)\right]=0 \\
& \therefore f^{2}=-\frac{1}{(2 N+1)\left(\frac{1}{4}+\frac{1}{Z N}+\frac{1}{4 N^{2}}\right)}
\end{aligned}
$$

もし $|N| \gg \mid$ ならば

$$
f^{2} \div-\frac{2}{N}
$$

(4-19)を $\mathrm{N} \gg 1$ の埸合にかきな打すと

$$
e^{-4}+f^{2} \frac{N}{z} e^{-Z N \psi} \sim 0
$$

が小さいとして

$$
1-\psi+f^{2} \frac{N}{2}(1-2 N \psi) \sim 0
$$


すなわち

$$
1+\frac{N f^{2}}{2}-4\left(1+N^{2} f^{2}\right) \sim 0
$$

従つて、0次の項は、。にできるがせの一次の項はけすことができない。

ঋ=0の磁力線附近ではdrift がなく従つてcharge separationがないがすこしはな

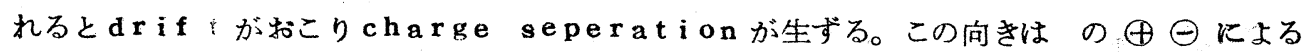
のでdipole 型ではなく、Quadrupole 型となる。

従つて、このような磁場では（4-15)を充すように $f$ をえらんで护けば di poleによる drift はなくなり、Quadrupole による变形がのこる。
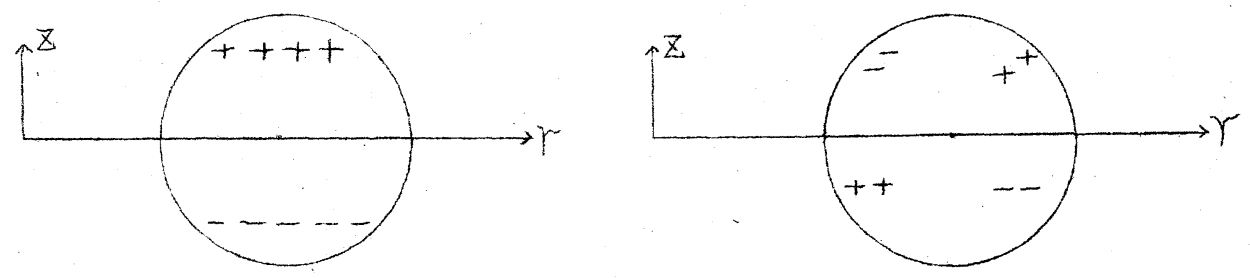

Tor us

p l asma 平衡の条件は

$$
B \phi=\frac{\hat{B}_{0} r_{0}}{r} \quad B \phi=\frac{B_{0} r_{0}}{r}\left[1+f\left(\frac{r}{r_{0}}\right)^{N} \cos N \phi\right]
$$

$$
\begin{aligned}
& \frac{\partial}{\partial 4} \phi \frac{d \Omega}{E^{2}}=0 \\
& \frac{1}{B^{2}}=e^{-2}\left(1-z f\left(1+\frac{1}{N}\right) e^{-N 4} \cos N \Omega+f^{2}\left\{\cos 2 N \Omega\left(\frac{4}{N}+\frac{1}{N^{2}}+4\right)+1+\frac{Z}{N}+\frac{1}{N^{2}}\right\} e^{-2 N 4}\right\} \\
& \frac{1}{2 \pi} \frac{\partial}{\partial \psi} f \frac{d \Omega}{B^{2}}=\frac{\partial}{\partial \psi}\left[e^{-2 \psi}+f^{z} e^{-z(N+1) \psi}\left(1+\frac{z}{N}+\frac{1}{N^{2}}\right)\right] \\
& =-2 E^{-24}-z(N+1) f^{2}\left(1+\frac{2}{N}+\frac{1}{N^{2}}\right) e^{-2(N+1) \psi} \quad(4-19)
\end{aligned}
$$

$\psi=0$ 近傍では(4-19)の0次のturnが0になつたとさに( アー9)は一燔さくなる。 即与

$$
1+(\mathrm{N}+1)\left(1+\frac{2}{\mathrm{~N}}+\frac{1}{\mathrm{~N}^{2}}\right) f^{2}=0
$$

N》1のときは

$$
f^{2} \doteqdot-\frac{1}{N}
$$


§5. 簡単な場合の直観的な取扱い

2 次元的に閉じられている磁力線を考光、これらは大部分円孤からなつているような簡単な場 合をとる。

先つ算初に図のように2つの section からなる un i t の 場合をとる。

section 1 と2では磁力線の向き は同じで曲率の向きが異るとする。 つなぎ目は充分短いとしとの影響は 無視する。

section 1, 2での磁場 $\mathrm{B}_{1}, \mathrm{~B}_{2}$ は

$$
\left\{\begin{array}{l}
B_{1}=B_{10} \frac{R_{1}}{r} \\
B_{2}=B_{20} \frac{R_{2}}{r}
\end{array}\right.
$$
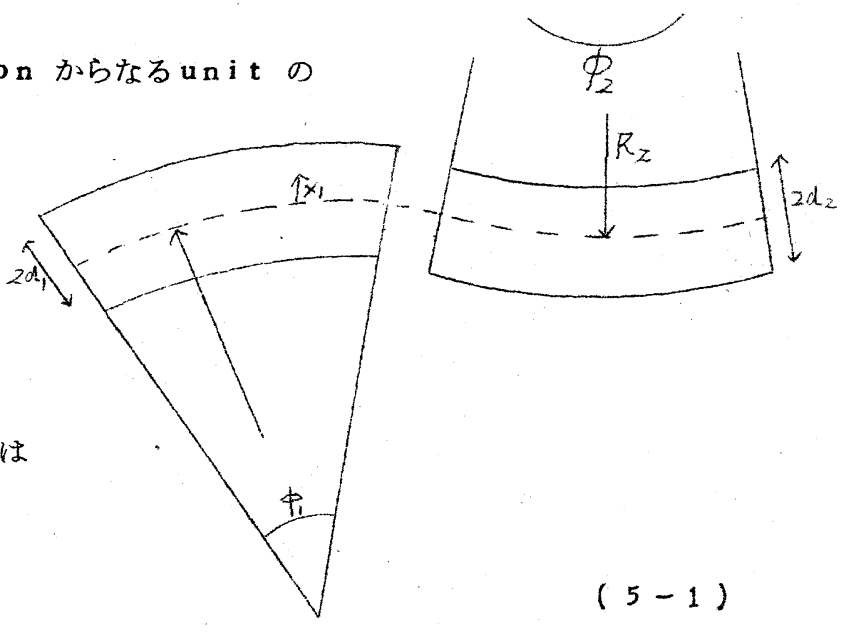

で与えられたとする。

$\mathrm{d}_{1}$ と $\mathrm{d}_{2}$ の関係は、それに含まれている $\mathrm{f} 1 \mathrm{u}$ 劝等しく、つなぎ目がなめらかにつながるよ5 にする。即ち

$$
\int_{R_{1}-d_{1}}^{R_{1}+d_{1}} B_{1} d r=\int_{R_{2}-d_{2}}^{\bar{x}_{2}+d_{2}} B_{2} d r
$$

$(5-2) \pm 5$

$$
\mathrm{B}_{10} \mathrm{R}_{1} \ell \mathrm{n} \frac{\mathrm{R}_{1}+\mathrm{d}_{1}}{\mathrm{R}_{1}-\mathrm{d}_{1}}=\mathrm{B}_{20} \mathrm{R}_{2} \ell \frac{\mathrm{R}_{2}+\mathrm{d}_{2}}{\mathrm{R}_{2}-\mathrm{d}_{2}}
$$

特に $\mathrm{R}_{1} \gg \mathrm{d}_{1}, \mathrm{R}_{2} \gg \mathrm{d}_{2}$ のときは

$$
\begin{aligned}
& \ln \frac{R_{1}+d_{1}}{R_{1}-d_{1}}=\frac{2 d_{1}}{R_{1}}+0 .\left(\frac{d_{1}^{3}}{R_{1}^{3}}\right) \text { をつかつて } \\
& B_{10} d_{1} \div B_{20} d_{2}
\end{aligned}
$$

が $\mathrm{d}_{1}$ と $\mathrm{d}_{2}$ の比を定めることになる。

ここでdriftを(1-9)をつかつて計算する。suffix 1，2はsection 1，2を示す。

$$
z_{D I}=\frac{m c \nu}{e} \int_{0}^{\phi_{1}} \frac{r_{1} d \phi}{B_{10} R_{1}}
$$




$$
\text { 大 河 } 千 \text { 弘 }
$$

中心線を基準にしてXで位置をきめることにすれば

$$
\mathrm{r}_{1}=\mathrm{R}_{1}+x_{1}
$$

$(5-5)$ は

$$
\begin{aligned}
& Z_{D_{1}}=\frac{m_{2} c v}{e} \frac{\phi_{1}}{B_{10}}\left(1+\frac{x_{1}}{R_{1}}\right) \\
& -Z_{D_{2}}=\frac{m c v}{e} \frac{\phi_{2}}{B_{20}}\left(1+\frac{x_{2}}{R_{2}}\right)
\end{aligned}
$$

ここで $x_{1}$ と $x_{2}$ は同じ磁力線の位置であるから

$$
x_{2} \approx \frac{d_{2}}{d_{1}} x_{1}
$$

故に 2 sections を通過した後の drift は

$$
\begin{aligned}
Z_{D} & =Z_{D_{1}}+Z_{D_{2}} \\
& =\frac{m c v}{e}\left[\frac{\phi_{1}}{B_{10}}-\frac{\phi_{2}}{B_{20}}+x\left\{\frac{\phi_{1}}{B_{1} R_{1}}+\frac{\phi_{2}}{B_{2} \cdot R_{1}} \frac{d_{2}}{d_{1}}\right\}\right](5-8)
\end{aligned}
$$

$x_{1}=0$ 即ち、中心線で $\mathrm{d} \mathbf{r}$ i f t $\mathrm{t}$ がなくなるだめはは

$$
\frac{\phi_{1}}{B_{10}}=\frac{\phi_{2}}{B_{20}}
$$

が充たされればよい。

ここでdipoleがなくなり、xのかかる頃は、Quadrupoleをあらわす。 (5-9)でB ${ }_{10}$ と B $_{20}$ を等しくとらなければ ${ }_{1}-\phi_{2}=0$ でこのunitをずつとつないでゆ けば Torus 状の放電管をつくることができる。

( 5ー9)はRを含まないので、Rは任意である。

p 1 a sma 平衡 即b

$$
j_{z} \cdot B_{p}=\frac{\partial p}{\partial r}
$$

をつかつて(3ー9)を計算すると

$$
\frac{\phi_{1}}{B_{i 0}^{2}}=\frac{\phi_{2}}{B_{20}}
$$

がえられる。

§6. Quadrupole を消すこと

§5のよ5な場合には、Quadrupole がのこるが、これを消すことを考える。 平面内では不可能であるから、Z方向にも磁力線をうねらすことにする。

図のように4つのsectionからなるunit を考える。 
Toroidal Magnet ic bottle
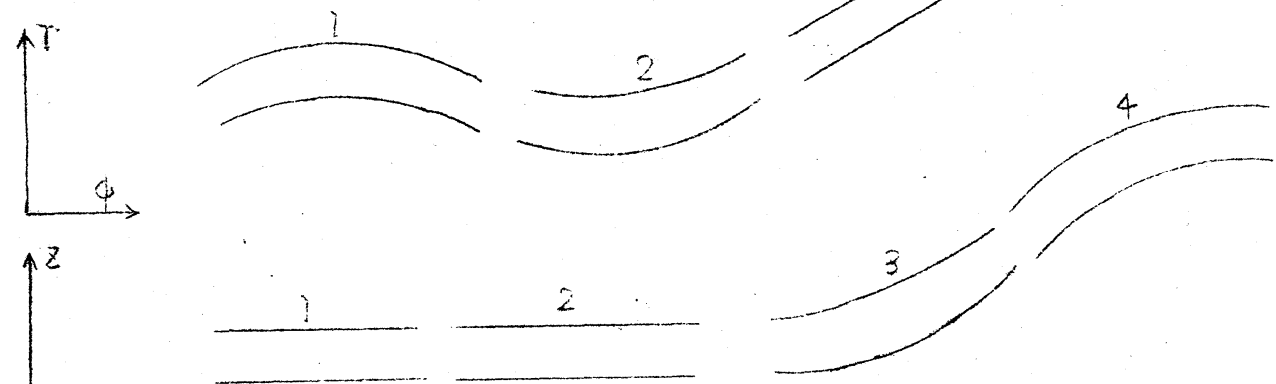

$\Phi$

section 1，2は、\$5のような sectionで 3，4はZ方向に円环でえがいてうねつて いる。磁力線の位置を知るために各 sect i onの断面は図のようであるとする。

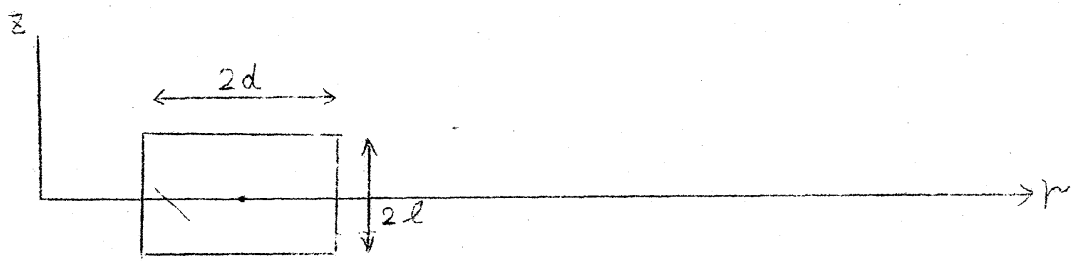

f 1 u $x$ 一定の条件は

$$
2 \ell_{1} \int_{R_{1}-d_{1}}^{R_{1}+d_{1}} B_{1} d r=2 \ell_{2} \int_{R_{2}-d_{2}}^{R_{2}+d_{2}} B_{2} d r=2 \ell_{3} \int_{R_{3}-d_{3}}^{R_{3}+d_{3}} B_{3} d r=2 l_{4} \int_{R_{4}-d_{4}}^{R_{4}+d_{4}} B_{4} d r \quad(6-1)
$$

即ち

$\ell_{1} B_{0} R_{1} \ln \frac{R_{1}+d_{1}}{R_{1}-d_{1}}=\ell_{2} B_{20} R_{2} \ln \frac{R_{2}+d_{2}}{R_{2}-d_{2}}=\ell_{3} B_{30} R_{3} \ln \frac{R_{3}+d_{3}}{\bar{R}_{3}-d_{3}}=\ell_{4} B_{40} R_{4} \ln \frac{R_{4}+d_{4}}{R_{4}-d_{4}}$ $\mathrm{R} \gg \mathrm{d}, \mathrm{R} \gg \ell$ の場合には

$$
\ell_{1} \mathrm{~B}_{10} \mathrm{~d}_{1} \doteqdot \ell_{2} \mathrm{~B}_{20} \mathrm{~d}_{2} \div \ell_{3} \mathrm{~B}_{30} \mathrm{~d}_{3} \doteqdot \ell_{4} \mathrm{~B}_{40} \mathrm{~d}_{4}
$$

次炕各 sectionで受けるdriftを計算する。

sect i on 1 K $x_{0}, Z_{0}$ なる初めの位置で粒子が入射したとする。

そのときにsection(1)で受けるdriftは

$$
Z_{D_{1}}=\frac{m C v}{l} \frac{\phi_{1}}{B_{1}}\left(1+\frac{x_{0}}{R_{1}}\right)
$$

従つて section（1）を出たときの座標は

$$
\begin{aligned}
& x_{1}=x_{0}, \\
& z_{1}=z_{0}+\frac{m c v}{l} \frac{\Phi_{1}}{B_{1}}\left(1+\frac{x_{0}}{R_{1}}\right)
\end{aligned}
$$


section (2) שはいると、先つ $x$ 㭫標が磁力線によつて $x_{1}$ から $\frac{\mathrm{d}_{2}}{\mathrm{~d}_{1}} x_{1}$ に移り、 $\ell_{1}=\ell_{2}$ とすればZはかわらない。

従つて section（2）をでるときには

$$
\begin{aligned}
& x_{2}=\frac{\mathrm{d}_{2}}{\mathrm{~d}_{1}} x_{1} \\
& \mathrm{z}_{2}=\mathrm{z}_{0}+\frac{m c v}{e}\left\{\left(\frac{\phi_{1}}{B_{1}}-\frac{\phi_{2}}{B_{2}}\right)+\left(\frac{\phi_{1} x_{0}}{B_{1} R_{1}}+\frac{\phi_{2} x_{1} d_{2}}{B_{2} R_{2} d_{1}}\right)\right\}
\end{aligned}
$$

section（3）にはいると、同じdriftの式をつからためには、 d r ift $+x$ の方向か $-Z$ の方向にかわる。したがつて（3）にはいつたときには

$$
\begin{aligned}
& x_{2} \text { は } \frac{\ell_{3} x_{2}}{\mathrm{~d}_{2}} \text { に移り、 } \\
& z_{2} \text { は } \frac{\mathrm{d}_{3}}{\ell_{2}} \mathrm{z}_{2} \text { に移る。 }
\end{aligned}
$$

$\mathrm{d} \mathbf{r}$ if $\mathrm{t}$ は $x$ 方向に执こる。従つて

$$
\left\{\begin{array}{l}
\dot{x}_{3}=\frac{\ell_{3}}{\mathrm{~d}_{2}} x_{2}+\frac{\operatorname{mev}}{e} \frac{\phi_{3}}{\mathrm{~B}_{3}}\left(1-\frac{1}{\mathrm{R}_{3}} \frac{\mathrm{d}_{3}}{\mathrm{~d}_{2}} \mathrm{z}_{2}\right) \\
\mathrm{z}_{3}=\frac{\mathrm{d}_{3}}{\ell_{2}} \mathrm{z}_{2}
\end{array}\right.
$$

section (4)にはいると

$x_{3}$ は $\frac{\ell_{4}}{\ell_{3}} x_{3}$ に

$z_{3}$ は $\frac{d_{4}}{d_{3}} z_{3}$ Kつる

drift

$$
\left\{\begin{array}{l}
x_{4}=\frac{l_{4}}{l_{3}} x_{3}-\frac{m c v}{e} \frac{\phi_{4}}{B_{4}}\left(1+\frac{1}{R_{4}} \frac{d_{4}}{d_{3}} z_{3}\right) \\
Z_{4}=\frac{d_{4}}{d_{3}} z_{3}
\end{array}\right.
$$

これが section（1）にはいるときには

$$
\left\{\begin{array}{l}
x=\frac{d_{1}}{l_{4}} x_{4} \\
z=\frac{l_{1}}{d_{4}} z_{4}
\end{array}\right.
$$

となる。

$x, Z$ Z $x_{0}, Z_{0}$ であらわすのであるが 


\section{Toroidal Magnet ic bottle}

今、特に

$$
\begin{aligned}
& \mathrm{B}_{10}=\mathrm{B}_{30}=\mathrm{B}_{40} \\
& \ell_{1}=\ell_{2}=\ell_{3}=\ell_{4}=\mathrm{d}_{1}=\mathrm{d}_{3}=\mathrm{d}_{4}
\end{aligned}
$$

従つて、, $B_{10} d_{1}=B_{20} d_{2}$ とし かつ、 s e c t i on (1) と（2），(3) と（4）の組は、夫々 (5-9)の条件を充すとする。

即ち、

そのとき

$$
\left\{\begin{array}{l}
\frac{\phi_{1}}{B_{10}}=\frac{\phi_{2}}{B_{20}} \\
\frac{\phi_{3}}{B_{30}}=\frac{\phi_{4}}{B_{40}}
\end{array}\right.
$$

$$
\left\{\begin{aligned}
x=x_{0}\left[1-\frac{(m c v)^{2}}{e^{2}}\left(\frac{\phi_{1}}{B_{10}} \frac{1}{R_{1}}+\frac{\phi_{2}}{B_{20}} \frac{1}{R_{1}} \frac{d_{2}}{d_{1}}\right)\left(\frac{\phi_{3}}{B_{30}} \frac{1}{R_{3}}+\frac{\phi_{4}}{B_{40} R_{4}}\right)\right] \\
\quad+Z_{0}\left[-\frac{m c v}{e}\left(\frac{\phi_{3}}{B_{30} R_{3}}+\frac{\phi_{4}}{B_{40} R_{4}}\right)\right] \quad(6-12) \\
Z=x_{0}\left(\frac{\phi_{1}}{B_{10} R_{1}}+\frac{\phi_{2}}{B_{2} R_{2}} \frac{d_{2}}{d_{1}}\right)+Z_{0}
\end{aligned}\right.
$$

matrixでかきなおせば

$$
\left(\begin{array}{l}
x \\
z
\end{array}\right)=\left(\begin{array}{cc}
1-b c & -b \\
c & 1
\end{array}\right)\left(\begin{array}{l}
x_{0} \\
z_{0}
\end{array}\right)
$$

ここに

$$
\begin{aligned}
& b=-\frac{m c v}{e}\left(\frac{\phi_{3}}{B_{30} R_{3}}+\frac{\phi_{4}}{B_{40} R_{4}}\right) \\
& c=\frac{m c v}{e}\left(\frac{\phi_{1}}{B_{10} R_{1}}+\frac{\phi_{2}}{B_{2} R_{2}} \frac{d 2}{d_{1}}\right)
\end{aligned}
$$

mat rix $\left(\begin{array}{cc}1-b c, & -b \\ c & 1\end{array}\right)$ の絶対値は1であるから

この $\mathrm{traceが} 2$ とー2の間にあれば， $x$ ，恀数の un i t を通過した後もその大きさが振動 するのみでかわらない。

安定の条件は

$$
-2<2-b c<2
$$

かきなして

$$
4>\text { bc }>0
$$


特别の場合として

$$
\left\{\begin{array}{l}
\oint_{1}=\oint_{3}=\oint_{4}=\oint_{0} \\
\mathrm{R}_{1}=\mathrm{R}_{3}=\mathrm{R}_{4}=\mathrm{R}_{0} \\
\mathrm{R}_{2} \frac{\mathrm{d}_{1}}{\mathrm{~d}_{2}}=\mathrm{R}_{0} \\
\mathrm{~B}_{10}=\mathrm{B}_{30}=\mathrm{B}_{40}=\mathrm{B}_{0}
\end{array}\right.
$$

ととれば、

$$
\begin{aligned}
& b=\frac{2 m c v \phi_{0}}{\ell B_{0} R_{0}} \\
& c=\frac{2 m c v \phi_{0}}{l B_{0} R_{0}}
\end{aligned}
$$

従つて安定条件 $(6-15)$ は

$$
4>4\left(\frac{m c \phi_{0}}{\ell B_{0} R_{0}}\right)^{2}>0
$$

即佔

$$
\left|\frac{m c v \phi_{0}}{e B \cdot R_{0}}\right|<1
$$

粒子のgyro-radius を ととすれば

$$
a=\frac{m c v}{e B_{0}}
$$

であるから (6-17) は

$$
\frac{a}{R_{0}} \phi_{0}<1
$$

となる。

この $\mathrm{d} \mathbf{r} \mathbf{i f t}$ の計算では、はじめから

$$
\frac{a}{R_{0}} \ll 1
$$

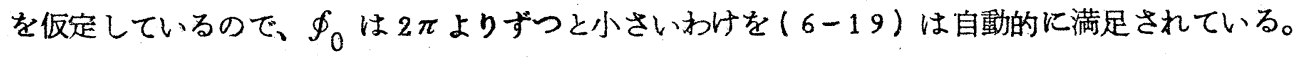
s e c t i on (1),(2)，(3)，(4)の配值ではＺ方向に放電管がずれるので不便であるから、 uni t としては（3）と（4)を半分づつに分けて
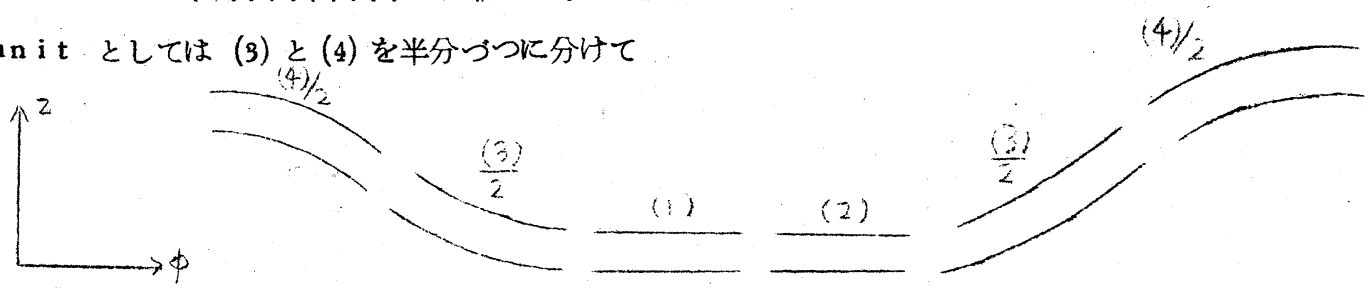
Toroidal Magnetic bottle

にするのが便利である。

plasma 平衙の条件は少し違うが、これは例えば section（2）の磁場を少し変えることに より、どららの条件をも実験的にためすことができる。

又、上の例では、平面内と Z方向とに磁力線が振動しているわけであるが、これをもつと連続的 に helical 磁力線にして放電管をそれに沿つてつくつてもよい。この場合は、 stellaratorの rotationa1 transformされた磁場のof $\mathrm{f}$ axisの磁力線をつかうこ とと同じであろう。 